\title{
DISCLOSURE VIA WEBSITE E AS CARACTERÍSTICAS DAS EMPRESAS DO TERCEIRO SETOR: UM ESTUDO EMPÍRICO DAS ENTIDADES CARIOCAS ${ }^{1}$
}

\author{
Carla Macedo Velloso dos Santos ${ }^{2}$ \\ Pierre Ohayon ${ }^{3}$ \\ Márcio Marvila Pimenta ${ }^{4}$
}

Resumo: O disclosure é tido como uma das principais formas de comunicação entre a empresa e os diversos usuários interessados em avaliar seu desempenho. Diante dessa realidade, destaca-se a Internet como um meio de comunicação com potencialidade de disponibilizar informações de forma tempestiva e transparente. Assim sendo, primeiramente, esta pesquisa objetivou examinar o nível de disclosure voluntário de informações financeiras e não financeiras de entidades do Terceiro Setor da cidade do Rio de Janeiro, por meio do Índice de Disclosure via Website e índices parciais, desenvolvidos com base na literatura. A amostra foi composta por 61 associações certificadas como Entidades de Utilidade Pública Federal (UPF) e como Entidades Beneficentes de Assistência Social (CEBAS). Após o exame, a pesquisa procurou identificar a existência de associação entre o referido nível e as características das entidades estudadas. Diante dos resultados, pode-se concluir que as entidades pouco se utilizam dos benefícios da Internet para a divulgação de suas informações, já que apresentaram níveis muito baixos de disclosure, principalmente quanto a informações financeiras e governança. Por meio de regressões, verificou-se associação positiva entre as receitas por doações e o nível de disclosure, o que pode indicar o uso do website para autopromoção diante de potenciais doadores. Ademais, verificou-se associação negativa entre o tempo da entidade como de Utilidade Pública Federal e o nível de disclosure, o que pode demonstrar preocupação das organizações mais recentes em construir uma boa reputação. Tal fato pode indicar, ainda, uma tendência à inércia na administração das organizações mais antigas. Por fim, os achados reforçam a necessidade de maior exigência por parte dos stakeholders e conscientização dos gestores das entidades do terceiro setor para que haja maior transparência e melhor comunicação via website, facilitando o controle social.

Palavras-chave: Disclosure; Website; Características; Terceiro Setor.

\footnotetext{
Trabalho apresentado na Sessão Especial do X Congresso Anpcont.

e-mail: carla.velloso.ufam@gmail.com. Universidade Federal do Amazonas.

${ }^{3}$ e-mail: pohayon@facc.ufrj.br. Universidade Federal do Rio de Janeiro.

${ }^{4}$ e-mail:mpimenta85@gmail.com. Universidade Federal Fluminense. 


\title{
DISCLOSURE VIA WEBSITE AND THE CHARACTERISTICS OF THE THIRD SECTOR ORGANIZATIONS: EMPIRICAL ANALYSIS OF ENTITIES FROM RIO DE JANEIRO
}

\begin{abstract}
The disclosure is regarded as one of the main forms of communication between the company and the various users interested in evaluating their performance. Given this reality, there is the Internet as a means of communication with the potential to provide information in a timely and transparent manner. First, this study aimed to examine the level of voluntary disclosure of financial and non financial informations of the third sector organizations from Rio de Janeiro City, through the Disclosure Index via Website and partial indexes, developed based on the literature. The sample was composed of 61 associations certified as Entities of Federal Public Utility and Charitable Entities of Social Assistance. After examination, the survey sought to identify the existence of an association between that level and the characteristics of the studied entities. Given the results, we can conclude that few entities use the benefits of the Internet to disseminate their information with very low levels of disclosure, particularly regarding the financial reporting and governance. Through regressions, there was a positive association between income from donations and the level of disclosure, which may indicate the use of the website for self-promotion to potential donors. In addition, there was a negative association between the entity's time as a Federal Public Utility and the level of disclosure, which can show concern of the youngest organizations to build a good reputation. This may also indicate a tendency to inertia in the administration of the oldest organizations. Finally, the findings reinforce the need for greater demand from stakeholders and awareness of managers of third sector organizations for greater transparency and better communication via website, facilitating citizen engagement.
\end{abstract}

Keywords: Disclosure; Website; Characteristics; Third sector. 


\section{INTRODUÇÃO}

mportante para a redução da assimetria informacional existente entre as empresas e seus stakeholders, o disclosure é tido como um dos requisitos da boa governança corporativa, sendo uma das principais formas de comunicação entre a empresa e os diversos usuários interessados em avaliar seu desempenho (Mendes-da-Silva, Ferraz-Andrade, Famá \& Filho, 2009, p. 191; Lima \& Pereira, 2011, p. 24). Este fato é observado tanto nas empresas com fins lucrativos como nas empresas do chamado Terceiro Setor, que é o "conjunto de organizações e iniciativas privadas que visam a produção de bens e serviços públicos que não geram lucro, mas respondem a necessidades coletivas" (Mañas \& Medeiros, 2012, p. 18).

Essas empresas, também chamadas de entidades sem fins lucrativos, geralmente possuem recursos financeiros advindos do governo e de doações de pessoas jurídicas e físicas (Behn, DeVries, \& Lin, 2010, p. 7), o que faz com que o disclosure seja necessário para que haja credibilidade diante de seus stakeholders: beneficiários, doadores, voluntários, governo, comunidade, entre outros interessados (Guo, 2007, p. 459; Gandia, 2011, p. 59; Saxton \& Guo, 2011, p. 273; Saxton, Kuo, \& Ho, 2012, p. 7). Nesse sentido, Behn, Devries e Lin (2010, p. 9) destacam a importância do disclosure para o aumento do fluxo de caixa dessas organizações, já que a divulgação de informações é um dos fatores que influenciam nas decisões dos doadores.

Diante dessa realidade, destaca-se a Internet como um meio de comunicação com potencialidade de disponibilizar essas informações de forma tempestiva e transparente, possuindo um custo relativamente baixo e acesso múltiplo e massivo, em comparação com relatórios impressos tradicionais (Cruz, Ferreira, Silva, \& Macedo, 2012, p. 155; Van Hulle \& Dewaelheyns, 2014, p. 69; Liket \& Maas, 2015, p. 278). Apesar de não haver obrigatoriedade, a entidade pode fazer uso das diversas funções interativas que a Internet permite para a divulgação de informações não financeiras e para estreitar o contato com seus stakeholders via website, como a divulgação de fotos e vídeos das ações realizadas e a manutenção de um espaço para críticas, sugestões e comentários dos usuários, entre outros recursos (Waters, 2007, p. 63).

Diversas pesquisas brasileiras focaram o disclosure via website das empresas com fins lucrativos, como é o caso dos estudos de Mendes-da-Silva et al. (2009, p. 190), Mendes-Da-Silva e Onusic (2014, p. 54) e Angonese, Sanches e Bezerra (2014, p. 19). Essas pesquisas, além de examinar o nível de disclosure via website de empresas de capital aberto, também verificaram a existência de associação entre o disclosure e as características das empresas, com o intuito de um melhor entendimento sobre os determinantes da divulgação voluntária de informações financeiras e não financeiras nas empresas estudadas.

No entanto, foram encontradas poucas pesquisas similares no Brasil voltadas às entidades sem fins lucrativos. Esse fato corrobora com os estudos de Nunes e Oliveira (2004, p. 3) e Olak, Slomski e Alves (2008, p. 25), que ressaltaram que pesquisas voltadas ao terceiro setor ainda são embrionárias no país. Além disso, Gandia (2011, p. 58) ressalta a importância de pesquisas que examinem a relevância da Internet na melhoria da transparência das entidades do terceiro setor, já que seus resultados podem indicar a necessidade da criação de códigos de boas práticas.

Sendo assim, surge a seguinte questão de pesquisa: Qual o nível de disclosure via Website de informações financeiras e não financeiras das entidades do Terceiro Setor e quais de suas características estão associadas com o referido nível? Para responder esta questão, primeiramente a pesquisa objetiva examinar o nível de disclosure voluntário de informações financeiras e não financeiras das entidades 
do Terceiro Setor, por meio do Índice de Disclosure via Website. Após esse exame, a pesquisa objetiva identificar a existência de associação entre o referido nível e as características das entidades estudadas.

Para a realização do estudo, foi utilizada uma amostra composta por 61 entidades certificadas como de Utilidade Pública Federal (UPF) e como Entidades Beneficentes de Assistência Social (CEBAS), localizadas na cidade do Rio de Janeiro, que ocupa a segunda posição dentre as capitais brasileiras em número de entidades como UPF.

Os achados desta pesquisa contribuem para a literatura sobre o tema com o fortalecimento das evidências sobre a associação entre o disclosure e as seguintes variáveis: porte, receitas por doações, receitas governamentais, rentabilidade e tempo de certificação como UPF. Além disso, espera-se que o Índice de Disclosure via Website utilizado no estudo possa ser usado por entidades do terceiro setor para a estruturação e melhoria de seus websites.

Este artigo está organizado em seis partes, incluindo esta introdução. Na segunda seção, é apresentada a literatura relevante acerca do tema. Em seguida, na terceira seção, são detalhadas as hipóteses testadas. Na quarta seção é relatada a metodologia adotada para a realização da pesquisa e na quinta seção são apresentados e discutidos os resultados empíricos alcançados no estudo. Por fim, na sexta seção, são apresentadas as considerações finais.

\section{REFERENCIAL TEÓRICO}

\subsection{DISCLOSURE VIA WEBSITE DAS ENTIDADES DO TERCEIRO SETOR}

Enquanto as entidades do terceiro setor não tiverem obrigatoriedade em divulgar suas ações e prestações de contas por meio de seus websites, os mesmos tornam-se objeto on-line de análise da importância dada pelas entidades à governança, disclosure voluntário, prestação de contas, comunicação com os stakeholders, entre outros fatores (Dumont, 2013, p. 1051). Sendo assim, diversas pesquisas internacionais foram realizadas com esse objetivo.

Com o intuito de explorar os websites de organizações sem fins lucrativos para avaliar o quanto tais entidades utilizam a Internet para atingir seus objetivos organizacionais, Kang e Norton (2004, p. 279) utilizaram como amostra as 100 maiores organizações dos Estados Unidos e constataram que os websites eram utilizados efetivamente para promover a boa imagem das entidades perante ao público. No entanto, os autores concluíram que a comunicação interativa com a sociedade encontrava-se insuficiente nos websites.

Por meio de análise de conteúdo, Ingenhoff e Koelling (2009, p. 66) analisaram o website de 134 entidades suíças de caridade com o objetivo de explorar seu potencial de comunicação com o público. Os resultados indicaram que o potencial da Internet para comunicação dialógica não foi utilizado de forma eficiente pela maioria das organizações de caridade. No entanto, os autores perceberam um certo reconhecimento da importância da comunicação, já que a maioria deles respondeu os pedidos de informação de potenciais doadores.

Ressaltando a importância da Internet para a comunicação efetiva e a promoção das entidades sem fins lucrativos junto aos doadores, voluntários, mídia e agências de fomento, Jamieson (2009, p. 23) escreveu um ensaio teórico onde descreve os passos que devem ser dados para transformar o website em uma ferramenta para atrair os stakeholders. Segundo o autor, o website deve ser interessante, dinâmico, popular e deve promover a efetiva comunicação com a comunidade. 
O estudo de Saxton e Guo (2011, p. 270) teve como objetivo principal verificar o uso da Internet para promover a prestação de contas, capacidade, governança e meio ambiente de 117 fundações comunitárias dos Estados Unidos. Diante dos resultados, os autores concluíram que os websites eram mais eficazes quando usados para fornecer divulgações financeiras do que para fornecer mecanismos de comunicação com as partes interessadas. Usando a análise multivariada, foram identificados o tamanho dos ativos e desempenho do conselho como os fatores mais importantes associados com a adoção de práticas de prestação de contas via website das entidades estudadas.

Focando na prestação de contas e transparência das organizações sem fins lucrativos da Espanha, Gandia (2011, p. 57) propôs um modelo de divulgação de informações para os websites, podendo servir como um guia para melhorar a transparência informativa e prestação de contas. Após análise empírica com 80 organizações, o autor revelou que os websites eram, fundamentalmente, ornamentais e que os níveis de divulgação estavam relacionados com a quantidade de futuras doações recebidas pelas organizações.

Em seu estudo, Dumont (2013, p. 1049), por meio de análise fatorial exploratória, desenvolveu o Índice "Nonprofit Virtual Accountability" como ferramenta para que organizações sem fins lucrativos possam planejar estrategicamente sua prestação de contas on-line, bem como para que os stakeholders possam analisar empiricamente os websites das organizações. Os componentes de prestação de contas virtuais sem fins lucrativos identificados durante a análise fatorial foram a acessibilidade, envolvimento, desempenho, governança e missão. Para testar a validade do instrumento, a autora aplicou-o em 468 organizações de Illinois, Estados Unidos, o que confirmou sua validade como uma ferramenta para medir a prestação de contas das entidades do terceiro setor.

Com o objetivo de medir a divulgação de informações de entidades do terceiro setor via website, Van Hulle e Dewaelheyns (2014, p. 69) analisaram 165 entidades belgas. Diante dos resultados, os autores concluíram que haviam semelhanças com os achados de pesquisas que focaram empresas com fins lucrativos, com exceção das informações financeiras e objetivos, que obtiveram níveis mais baixos de divulgação nas entidades do terceiro setor. Ademais, os autores concluíram que pressões impostas pelos stakeholders, como previsto pela teoria neo-institucional, pode facilmente melhorar o nível de divulgação das organizações, da mesma forma que empresas com fins lucrativos atendem seus clientes.

Nota-se que o tema tem sido foco de pesquisas em diversos países. No Brasil ainda é incipiente o tema, principalmente nos estudos junto a entidades do terceiro setor (Olak, Slomski, \& Alves, 2008, p. 25). No entanto, destaca-se a pesquisa de Flach, Rosa, e Stupp $(2014$, p. 3) que teve como objetivo analisar o nível de transparência online de organizações do terceiro setor na Região Sul do Brasil. Com a amostra de 50 organizações de cada estado, foi aplicado um levantamento de informações com o uso de um questionário elaborado com base na importância organizacional e contábil. Com os resultados, os pesquisadores concluíram que o nível de evidenciação é relativamente baixo, reforçando a necessidade de desenvolver ferramentas mais eficientes e acessíveis de busca e obtenção de informações dessas organizações, além da necessidade de maior normatização e fiscalização no setor.

\section{HIPÓTESES}

Nesta seção, serão abordados achados de pesquisas nacionais e internacionais que apontam associações entre algumas características das empresas, com ou sem fins lucrativos, com seus níveis de divulgação voluntária de informações relevantes para a tomada de decisão. Diante desses achados, 
serão apresentadas as hipóteses que consideram que algumas dessas características podem influenciar no disclosure via website das entidades certificadas como Utilidade Pública Federal da cidade do Rio de Janeiro.

\subsection{PORTE DA ENTIDADE}

A literatura fornece argumentos que defendem que o porte da empresa pode influenciar na forma que suas informações são divulgadas aos seus stakeholders, já que devido a maior complexidade organizacional, as empresas de maior porte tendem a adotar práticas de gestão mais profissionais (Mendes-da-Silva et al., 2009, p. 193).

Além disso, Saxton e Guo (2011, p. 285) ressaltam que entidades sem fins lucrativos de maior porte possuem capacidade tecnológica mais avançada que as entidades menores, o que favorece a divulgação de suas informações via website.

Estudos como de Dumont (2013, p. 1049), Mendes-da-Silva et al. (2009, p. 190) e Mendes-Da-Silva e Onusic (2014, p. 54) corroboram com essas afirmativas, já que concluíram que quanto maior o porte das empresas estudadas, maior o volume de informações divulgadas via website. Sendo assim, surge a primeira hipótese desta pesquisa:

H1: O porte é uma característica que influencia positivamente na quantidade de informações divulgadas pelas entidades do terceiro setor via website.

\subsection{FONTE DE RECURSOS}

A segunda e a terceira hipótese da pesquisa referem-se a duas das fontes de recursos da entidade: a) receitas por doações de pessoas físicas ou jurídicas e b) receitas governamentais.

Segundo os estudos internacionais de Saxton e Guo (2011, p. 279) e Saxton et al. (2012, p. 1057), entidades sem fins lucrativos que possuem receitas predominantemente advindas de doações adotam um maior nível de divulgação de informações relevantes via website, já que o nível de transparência pode influenciar no volume das doações.

Outros estudos ratificam esses achados, como a pesquisa de Tinkelman (1999, p. 135) que afirma que o volume de doações é afetado pela qualidade das informações da entidade. Greenlee e Brown (1999, p. 111) explicam que a qualidade das informações está intimamente ligada à sua disponibilidade, evidenciando que o disclosure voluntário auxilia os potenciais doadores à tomada de decisão. Também Trussel e Parsons (2007, p. 283) demonstraram em seu estudo que a disponibilidade de informações a partir de relatórios financeiros de entidades do terceiro setor é um importante determinante de suas doações.

Segundo Zhuang, Saxton e Wu (2014, p. 469), a divulgação de informações financeiras, de desempenho, de dados relacionados à captação de recursos e a relação com doadores é, portanto, uma ferramenta importante para organizações do terceiro setor que tentam atrair maiores doações, aumentando, ao mesmo tempo, a accountability e a confiança dos stakeholders. Diante disso, a segunda hipótese deste estudo é:

H2: Maior proporção de receita por doações é uma característica que influencia positivamente na quantidade de informações divulgadas pelas entidades do terceiro setor via website. 
Já Lima e Pereira (2011, p. 36) concluíram que o volume de recursos recebidos por governos federais, estaduais e municipais ou de empresas públicas influencia significativamente no nível de disclosure das instituições filantrópicas de ensino superior do Brasil. Para Borges, Paulo e Pereira (2014, p. 14) as entidades que possuem receitas predominantemente advindas de subvenções públicas possuem um desempenho operacional melhor que as demais, podendo levar a uma melhor divulgação voluntária de informações.

Apesar de pouca evidência em estudos anteriores, optou-se por analisar a influência das receitas advindas por subvenções governamentais no nível de disclosure das entidades da amostra. Assim, a terceira hipótese deste estudo é:

H3: Maior proporção de receita governamental é uma característica que influencia positivamente na quantidade de informações divulgadas pelas entidades do terceiro setor via website.

\subsection{RENTABILIDADE}

Campos, Moreira e Scalzer (2014, p. 137) consideram que os gestores de entidades do terceiro setor com melhor rentabilidade têm incentivos para demonstrar a sua capacidade de retorno à sociedade pois, mesmo sem fins lucrativos, o superávit é um sinal de que a entidade será capaz de cumprir seus objetivos sociais.

Corroborando, Ávila (2013, p. 13) ressalta que há equívocos na interpretação do caráter não lucrativo, já que o superávit deve existir também nas organizações do terceiro setor para que seja possível o reinvestimento em suas atividades e a consecução de seus objetivos sociais. Sendo assim, seu estudo concluiu que existia relação positiva significativa entre a rentabilidade e a transparência da entidade estudada.

No entanto, para Saxton, Kuo e Ho (2012, p. 1058), a rentabilidade pode afetar as decisões relacionadas à divulgação das entidades do Terceiro Setor de forma inversa. Os autores citam o caso dos hospitais filantrópicos de Taiwan, que quando apresentam altos superávits tendem a não divulgar amplamente seus números para evitar reação desfavorável do público. Já hospitais com superávits mais baixos ou déficits tendem a disponibilizar suas demonstrações para conduzir a opinião pública a seu favor e, por sua vez, favorecer o aumento de sua arrecadação.

Diante dessa ambiguidade, surge a quarta hipótese:

H4: A rentabilidade é uma característica que influencia negativamente na quantidade de informações divulgadas pelas entidades do terceiro setor via website.

\subsection{TEMPO COMO UPF}

O estudo de Gandia (2011, p. 67) concluiu que a idade das entidades sem fins lucrativos da Espanha é uma característica que possui associação significativa com o disclosure via website. Para o autor, a reputação é construída ao longo dos anos e, devido a isso, entidades mais antigas tendem a divulgar suas informações com maior qualidade.

No entanto, algumas pesquisas discordam com essa visão. Saxton e Guo (2011, p. 277) defendem que as entidades sem fins lucrativos mais jovens tendem a ser mais inovadoras, sendo mais propensas a recorrerem aos websites para divulgar suas prestações de contas, diferentemente das entidades mais antigas, que tendem à inércia. 
Mendes-da-Silva et al. (2009, p. 194) e Mendes-Da-Silva e Onusic (2014, p. 60), que estudaram o disclosure via website das empresas de capital aberto, ressaltaram que as empresas mais recentemente listadas na bolsa de valores poderiam ter um maior incentivo em divulgar suas informações voluntariamente, para assim angariar a confiança dos investidores. Daí surge a inquietação se esse tipo de comportamento também seria observado em entidades mais recentemente certificadas com o título de UPF:

H5: Tempo como UPF é uma característica que influencia negativamente na quantidade de informações divulgadas pelas entidades do terceiro setor via website.

\section{METODOLOGIA}

\subsection{CLASSIFICAÇÃO DA PESQUISA}

Como existe pouco conhecimento e resultados sobre a questão de pesquisa no Brasil, o atual estudo pode ser classificado como exploratório, assim como as pesquisas de Behn, DeVries e Lin (2010, p. 7) e Marudas e Jacobs (2011, p. 2). A pesquisa também pode ser classificada como bibliográfica, com o uso de artigos científicos para embasar a estruturação do Índice de Disclosure via Website e a seção teórica.

A pesquisa também classifica-se como documental, já que foram levantados dados nos websites e nas prestações de contas eletrônicas das entidades, procedimento também realizado por Angonese Sanches e Bezerra (2014, p. 23) e Rezende, Facure e Dalmácio (2009, p. 2). Além disso, a pesquisa é classificada como quantitativa, já que faz uso de quantificação na coleta dos dados e tratamento estatístico (Angonese et al., 2014, p. 23).

\subsection{SELEÇÃO DA AMOSTRA}

No mês de julho de 2015, haviam 12.216 entidades do Terceiro Setor certificadas pelo Ministério da Justiça como Entidades de Utilidade Pública Federal (UPF) em todo o Brasil, sendo 7.111 na Região Sudeste, 2.738 na Região Sul, 1.289 na Região Nordeste, 865 na Região Centro-Oeste e 213 em toda Região Norte (CNEs/MJ).

Com base nas pesquisas de O'Regan e Oster (2002, p. 359) e Guo (2007, p. 458), que tiveram como escopo entidades sem fins lucrativos de grandes capitais americanas, optou-se por estudar as entidades da cidade do Rio de Janeiro, por ser esta a segunda capital do Brasil em número de entidades certificadas como UPF, além de serem escassos estudos abordando as entidades do terceiro setor dessa cidade, especificamente.

Por meio do Cadastro Nacional de Entidades de Utilidade Pública do Ministério da Justiça (CNEs/ MJ), foram localizadas 457 entidades na Cidade do Rio de Janeiro, incluindo associações e fundações. Para os cálculos estatísticos desta pesquisa, as informações contábeis do exercício de 2014 deveriam estar disponíveis no website do Ministério da Justiça. Contudo, apenas 191 entidades estavam com suas prestações de contas eletrônicas de 2014 aprovadas até o dia 21/07/2015, data da coleta das informações no CNEs/MJ. Adicionalmente, para uma maior homogeneidade, foram consideradas apenas as associações que possuíam o Certificado de Entidades Beneficentes de Assistência Social (CEBAS), o que resultou em uma amostra de 61 entidades. 


\section{3 ÍNDICE DE DISCLOSURE}

O índice de disclosure utilizado nesta pesquisa, denominado Índice de Disclosure via Website (I_DiscWeb), foi baseado nas informações solicitadas pelo Ministério da Justiça como forma de prestação de contas anual das entidades certificadas como UPF, conforme a Portaria SNJ n 23/2006 (Manual CNEs/ MJ, 2007, p. 143). Além desses dados, o I_DiscWeb foi composto por itens pertinentes das pesquisas de Gandia (2011, p. 63), Mendes-da-Silva et al. (2009, p. 195), Lima e Pereira (2011, p. 32 - 34), Milani Filho (2010, p. 6) e Dumont (2013, p. 1055).

Assim, o instrumento de coleta de dados utilizado nesta pesquisa, em forma de checklist, foi estruturado em 42 itens, organizados em três categorias baseadas no trabalho de Gandia (2011, p. 62). Dessa forma, foi possível a obtenção de três índices parciais, descritos na Tabela 1, e a junção desses três índices resultou no I_DiscWeb.

Tabela 1: Índices parciais para a obtenção do I_DiscWeb

\begin{tabular}{|c|c|c|}
\hline Código & Categorias & Descrição \\
\hline I_Ger & $\begin{array}{l}\text { Índice de } \\
\text { Informações } \\
\text { Gerais }\end{array}$ & $\begin{array}{l}\text { O website deve conter informações que promovam as atividades } \\
\text { realizadas pelas entidades, dando oportunidade aos stakeholders de } \\
\text { conhecerem como as entidades atuam na prática. Nesta categoria, } 14 \\
\text { itens foram incluídos, representando } 33 \% \text { do I_DiscWeb. O índice é } \\
\text { apresentado na forma de proporção, variando de } 0 \text {, quando nenhum } \\
\text { item é atendido, a 1, quando todos os itens são atendidos. }\end{array}$ \\
\hline I_FinGov & $\begin{array}{l}\text { Índice de } \\
\text { Informaçōes } \\
\text { Financeiras } \\
\text { e sobre } \\
\text { Governança }\end{array}$ & $\begin{array}{l}\text { O website deve conter informações financeiras e sobre governança, } \\
\text { que são essenciais para que os stakeholders possam conhecer a } \\
\text { situação econômica das entidades e possam julgar as ações dos } \\
\text { gestores e conselheiros. Nessa categoria, são considerados } 18 \text { itens, o } \\
\text { que representa } 43 \% \text { do I DiscWeb. O índice é apresentado na forma } \\
\text { de proporção, variando de } 0 \text {, quando nenhum item é atendido, a } 1 \text {, } \\
\text { quando todos os itens são atendidos. }\end{array}$ \\
\hline I_Nav & $\begin{array}{l}\text { Índice de } \\
\text { Apresentação } \\
\text { e Navegação } \\
\text { do website }\end{array}$ & $\begin{array}{l}\text { O website deve possuir uma apresentação clara, com condições } \\
\text { de acessibilidade e navegabilidade. } 10 \text { itens são analisados nessa } \\
\text { categoria, representando } 24 \% \text { do I_DiscWeb. O índice é apresentado } \\
\text { na forma de proporção, variando de } 0 \text {, quando nenhum item é } \\
\text { atendido, a } 1 \text { quando todos os itens são atendidos. }\end{array}$ \\
\hline
\end{tabular}

Fonte: Elaborado pelos autores, baseado em Gandía (2011, p. 62).

Para verificar a consistência interna dos índices, foi realizada análise de confiabilidade com base no alfa de Cronbach, assim como na pesquisa de Gandia (2011, p. 62). Segundo Hair, Black, Babin, Anderson e Tatham (2009, p. 126), este indicador varia entre zero e um e seu limite inferior geralmente aceito é 0,70. Os resultados obtidos foram os seguintes: I_Ger: 0,903; I_FinGov: 0,83; I_Nav: 0,765; e I_DiscWeb: 0,926 . Sendo assim, nota-se que os índices, tanto parcialmente como em conjunto, podem ser considerados consistentes.

Para a coleta, primeiramente, realizou-se a busca pelo endereço do website oficial de cada entidade no CNEs/MJ. A partir disso, os websites foram examinados com o uso do checklist durante os meses de agosto e setembro de 2015 e seus resultados tabulados em planilhas do Microsoft Excel.

Adotou-se a mesma escala de Milani Filho $(2010$, p. 8) para a pontuação de cada item, em que 0 (zero) representa a ausência e 1 (um) a presença da informação no website da entidade e nenhuma categoria apresentou peso diferenciado para o cálculo da pontuação final, já que o objetivo foi apenas quantificar as informações divulgadas nos websites das entidades do terceiro setor. O Índice de Disclosure via Website (I_DiscWeb) também é apresentado na forma de proporção, variando de 0, quando nenhum item é atendido, a 1, quando todos os itens são atendidos. Dessa forma, o I_DiscWeb pode ser representado pela seguinte expressão: I_DiscWeb $=\left(\left(I \_G e r .14\right)+(\right.$ I_FinGov.18) + (I_Nav.10))/42

4.4 TESTES ESTATÍSTICOS

Foram realizadas regressões simples para testar as hipóteses propostas na pesquisa com o uso do software Statistical Package for the Social Sciences - SPSS, sendo, primeiramente, testados e compro- 
vados os pressupostos das regressões (normalidade dos resíduos e homoscedasticidade dos resíduos) com o uso do software Gretl. Utilizou-se o Índice de Disclosure via Website (I_DiscWeb) como variável dependente e cinco variáveis independentes, uma para cada hipótese proposta, conforme demonstrado na Tabela 2. Tais variáveis foram coletadas da prestação de contas eletrônica do exercício de 2014 de cada entidade, disponíveis no CNEs/MJ.

Tabela 2: Variáveis independentes (explicativas) do Índice de Disclosure via Website

\begin{tabular}{|c|c|c|c|}
\hline Variáveis & Proxy & Código & Estudos anteriores que utilizaram a proxy \\
\hline Porte da Entidade & Logaritmo natural do total das receitas de 2014 & Port & Saxton, Kuo e Ho (2012) e Dumont (2013) \\
\hline Receitas de Doações & $\begin{array}{c}\text { Proporção das receitas de } 2014 \text { advindas de } \\
\text { doações }\end{array}$ & Rdoa & Saxton, Kuo e Ho (2012) e Saxton e Guo (2011) \\
\hline Receitas Governamentais & $\begin{array}{c}\text { Proporção das receitas de } 2014 \text { advindas do } \\
\text { governo }\end{array}$ & Rgov & $\begin{array}{l}\text { Borges, Paulo e Pereira (2014) e Lima e Pereira } \\
\text { (2011) }\end{array}$ \\
\hline Rentabilidade & Rentabilidade do Ativo Total de 2014 (ROA) & Rent & $\begin{array}{l}\text { Saxton, Kuo e Ho (2012), Ávila (2013) e (Campos } \\
\text { et al., 2014) }\end{array}$ \\
\hline Tempo como UPF & $\begin{array}{l}\text { Logaritmo natural do tempo, em anos, que a } \\
\text { entidade está certificada como UPF. Contagem } \\
\text { do tempo até } 2014 .\end{array}$ & Temp & $\begin{array}{c}\text { Mendes-da-Silva et al. (2009) e Mendes-da-Silva } \\
\text { e Onusic (2010) }\end{array}$ \\
\hline
\end{tabular}

As variáveis independentes foram defasadas em um período, assim como na pesquisa de Mendes-da-Silva et al. (2009, p. 190). Esse procedimento visa verificar se as características anteriores das entidades exercem influência sobre seu nível de disclosure atual.

Além das regressões, foi utilizada a estatística descritiva para a caracterização da amostra e o nível de Disclosure via Website, onde foram calculados a média, mediana e desvio padrão dos resultados.

\section{ANÁLISE DOS RESULTADOS}

5.1 PERFIL DA AMOSTRA

A amostra foi composta por 61 associações localizadas no Rio de Janeiro certificadas como Utilidade Pública Federal pelo Ministério da Justiça (MJ) e como Entidade Beneficente de Assistência Social pelo Ministério do Desenvolvimento Social e Combate à Fome (MDS). Dessas, 34\% eram entidades assistenciais, $28 \%$ beneficentes, $13 \%$ educacionais e os $25 \%$ restante eram entidades religiosas, classistas, hospitalar, cultural, entre outras.

A Tabela 3, a seguir, apresenta a estatística descritiva das características das entidades da amostra. Tais características serviram de base para as seguintes proxies utilizadas neste trabalho: Porte da Entidade; Receitas de Doações; Receitas Governamentais; Rentabilidade; e Tempo como UPF.

Tabela 3: Estatística descritiva das características das entidades da amostra - dados de 2014

\begin{tabular}{|c|c|c|c|c|}
\hline Características & Média & Mediana & Mínimo & Máximo \\
\hline Receita Bruta & $\mathrm{R} \$ 4.806 .871$ & $\mathrm{R} \$ 1.327 .249$ & $\mathrm{R} \$ 29.465$ & $\mathrm{R} \$ 37.355 .281$ \\
\hline Proporção das Receitas por doações & $45 \%$ & $37 \%$ & $0 \%$ & $100 \%$ \\
\hline Proporção das Receitas Governamentais & $20 \%$ & $0 \%$ & $0 \%$ & $100 \%$ \\
\hline Superávit/ Déficit do Exercício & $-R \$ 3.159$ & $\mathrm{R} \$ 2.375$ & $-\mathrm{R} \$ 12.156 .231$ & $\mathrm{R} \$ 11.901 .892$ \\
\hline Ativo Total & $\mathrm{R} \$ 12.319 .998$ & $\mathrm{R} \$ 1.253 .021$ & $\mathrm{R} \$ 19.773$ & $\mathrm{R} \$ 216.421 .057$ \\
\hline ROA & $-R \$ 0,11$ & $\mathrm{R} \$ 0$ & $-R \$ 3,58$ & $R \$ 0,51$ \\
\hline Anos como UPF & 25 & 18 & 1 & 57 \\
\hline
\end{tabular}

Fonte: Elaborado pelos autores com base nos dados do Ministério da Justiça (CNEs/MJ, 2015).

Quanto à receita bruta, verificou-se que existe uma grande discrepância entre a entidade "Obra de Assistência Social Gaspar da Silva Araújo", que apresentou a menor receita bruta e o "Centro de Integração Empresa-Escola do Rio de Janeiro", com a maior receita. 
Quanto à proporção das receitas advindas de doações, a média ficou em 45\%. Não obtiveram receitas por doações no ano de 2014 13\% das entidades da amostra, contudo 11\% tiveram 100\% de suas receitas advindas de doações.

Já em relação à proporção das receitas governamentais, a média da amostra ficou em 20\%. Das entidades analisadas, $56 \%$ não obtiveram receitas advindas do governo e apenas a entidade"Solazer - $O$ Clube dos Excepcionais" teve 100\% de suas receitas do ano de 2014 advindas de subvenções.

Em relação ao resultado, a média das entidades apresentou um déficit de $\mathrm{R} \$ 3.159$, quando a mediana apresentou um superávit de $\mathrm{R} \$ 2.375$. Um fato preocupante observado refere-se ao número de entidades que apresentaram déficit no ano de 2014, correspondendo a $46 \%$ da amostra. A "Associação Civil das Servas de Maria do Brasil" foi a entidade que apresentou o maior déficit da amostra e a"Real e Benemérita Sociedade Portuguesa Caixa de Socorros D. Pedro V" a que apresentou o maior superávit.

O ativo total de 2014 teve média de R\$12.319.998 entre as entidades da amostra. A entidade“Novo Rumo Obras Sociais" apresentou o menor ativo total, quanto que a"Província Brasileira da Congregação da Missão (Padres Lazaristas)" foi a entidade com o maior ativo.

Quanto ao Retorno sobre os Ativos (ROA), percebe-se um valor mínimo negativo de $\mathrm{R} \$ 3,58$ referente à entidade "Obra Social da Cidade do Rio de Janeiro", significando que a cada R\$ 1,00 investido em ativos, a entidade perdeu $\mathrm{R} \$ 3,58$ de seu resultado no ano de 2014. Já o valor máximo é de apenas R\$ 0,51, referente à entidade "Assessoria e Serviços a Projetos em Agricultura Alternativa", que a cada $\mathrm{R} \$ 1,00$ investido obteve $\mathrm{R} \$ 0,51$ de seu resultado no ano de 2014.

Em média, as entidades da amostra possuíam 25 anos como UPF em 2014. As entidades "Lar de Daniel Cristóvão" e"Sociedade Beneficente das Damas Israelitas do Rio de Janeiro" eram as mais recentes, possuindo apenas um ano. A "Sociedade Pestalozzi do Brasil", com 57 anos como UPF, foi a mais antiga da amostra analisada.

5.2 NÍVEL DE DISCLOSURE VIA WEBSITE

Após a coleta das informações financeiras e não financeiras das entidades da amostra e após a verificação dos instrumentos utilizados em seus websites para melhorar a navegabilidade e transparência, foi possível auferir o nível de disclosure via website dessas entidades, por meio do Índice de Disclosure via Website (I_DiscWeb), além dos índices parciais propostos nesta pesquisa. A Tabela 4 apresenta as estatísticas descritivas dos referidos índices.

Tabela 4: Estatística descritiva do Índice de Disclosure via Website e índices parciais

\begin{tabular}{|c|c|c|c|c|c|}
\hline Índices & Mínimo & Máximo Possível & Máximo Observado & Média & Desvio Padrão \\
\hline I_Ger & 0 & 1 & 0,857 & 0,436 & 0,286 \\
\hline I_FinGov & 0 & 1 & 0,722 & 0,069 & 0,123 \\
\hline I_Nav & 0 & 1 & 0,800 & 0,290 & 0,230 \\
\hline I_DiscWeb & 0 & 1 & 0,690 & 0,244 & 0,176 \\
\hline
\end{tabular}

A média dos índices mostraram-se baixas e com considerável dispersão, com destaque ao Índice de Informações Financeiras e sobre Governança, com média de apenas 0,069. Destaca-se, ainda, que 23\% das entidades da amostra não possuíam website, o que explica o fato de todos os índices apresentarem valores mínimos nulos e o que demonstra uma grande carência de transparência dessas entidades.

Tais fatos são observados com maior clareza por meio dos gráficos 1, 2, 3 e 4, que apresentam o histograma dos índices analisados: Índice de Informações Gerais; Índice de Informações Financeiras e sobre Governança; Índice de Apresentação e Navegação do Website; e Índice de Disclosure via Website, respectivamente. 

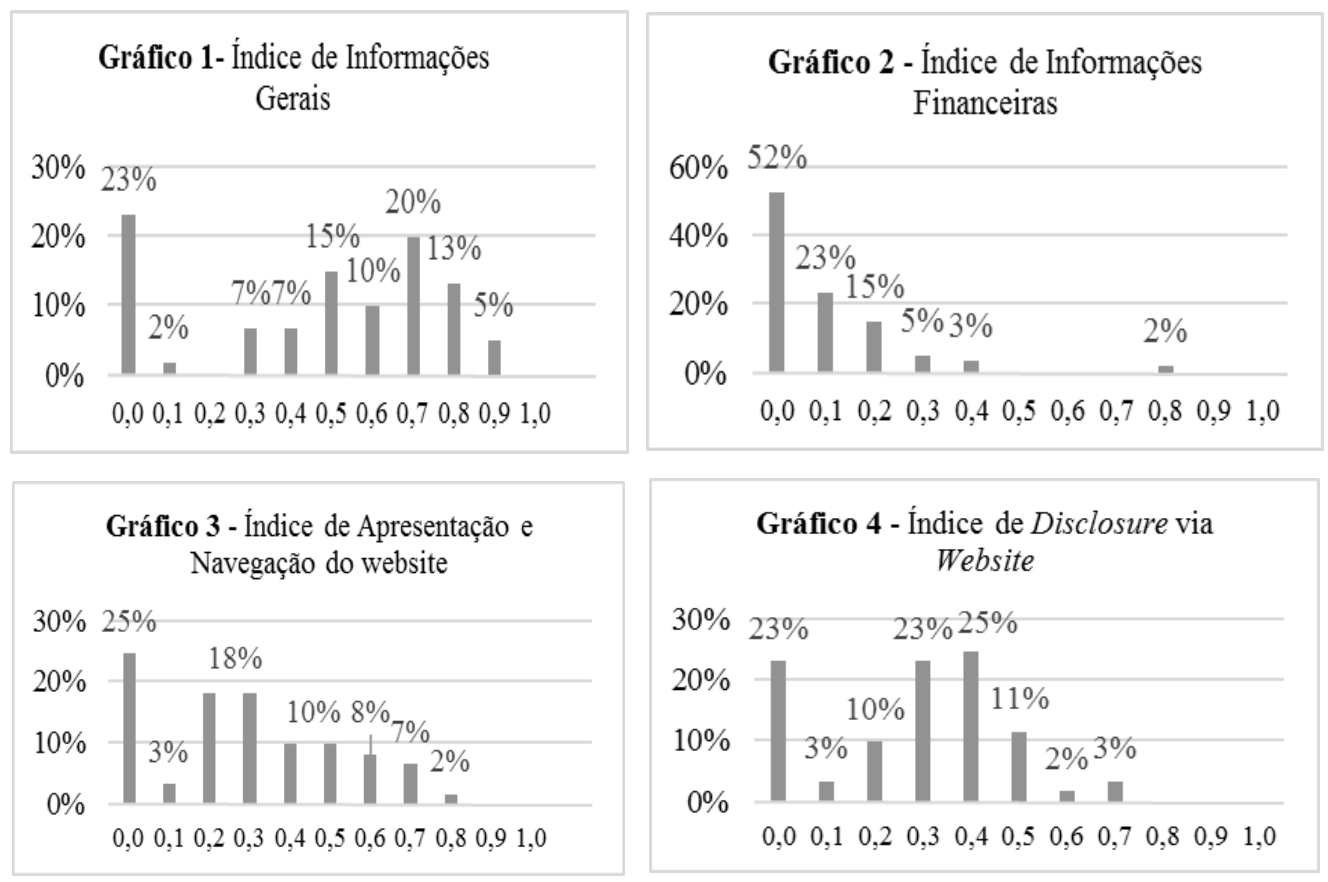

Fonte: Elaborado pelos autores com base na verificação dos 61 websites.

Ao observar o Gráfico 1, que se refere ao Índice de Informações Gerais, pode-se perceber que a maioria das entidades que possuíam sites atenderam mais de $50 \%$ dos itens do referido índice, apresentando um nível regular de evidenciação, similarmente à pesquisa de Dumont $(2013$, p. 1058) e Lima e Pereira (2011, p. 41). As entidades "Associação Vencer", "Banco da Providência” e "Casa de Francisco de Assis" destacaram-se por apresentar índice acima de 0,85.

O Gráfico 2 corresponde ao Índice de Informações Financeiras e Governança. Dentre os demais, esse foi o índice de menor atendimento, onde a grande maioria das entidades apresentou índices menores de 0,10 , correspondendo a menos de $10 \%$ das informações pesquisadas. Somente o "Instituto Brasileiro de Análises Sociais e Econômicas - IBASE" apresentou um bom resultado, com índice de 0,72, divulgando as informações financeiras do ano de 2014 e anos anteriores, parecer da auditoria, entre outros. Esses resultados assemelham-se aos achados de Milani Filho (2010, p. 12), Gandia (2011, p. 75), Lima e Pereira (2011, p. 41) e Dumont (2013, p. 1062).

Quanto ao Índice de Apresentação e Navegação do Website, o Gráfico 3 indica que a grande maioria das entidades obteve índices menores de 0,50, ou seja, fez uso de menos de $50 \%$ das ferramentas avaliadas, diferentemente dos resultados encontrados no estudo de Dumont (2013, p. 1060) quanto à acessibilidade dos websites. A entidade de maior destaque foi o "Instituto Ronald Mcdonald de Apoio à Criança", com índice de 0,80.

Finalmente, como pontuação geral, o Índice de Disclosure via Website é demonstrado no Gráfico 4. Mostra-se preocupante que a maioria das entidades apresentou índices menores de 0,40, ou seja, possuindo um atendimento de menos de $40 \%$ dos itens avaliados. Esse nível, muito aquém do desejado, dificulta o controle social e pode comprometer, inclusive, o volume das doações recebidas, de acordo com a pesquisa de Trussel e Parsons (2007, p. 282). Das entidades da amostra, destacam-se o "Instituto Ronald Mcdonald de Apoio à Criança", com índice de 0,57, a "Casa de Francisco de Assis", com índice 
de 0,62 e o "Instituto Brasileiro de Análises Sociais e Econômicas - IBASE", com índice de 0,69, melhor índice da amostra.

Quanto aos itens que compuseram cada um dos índices, os Gráficos 5, 6 e 7 demonstram a proporção de entidades que os utilizaram.

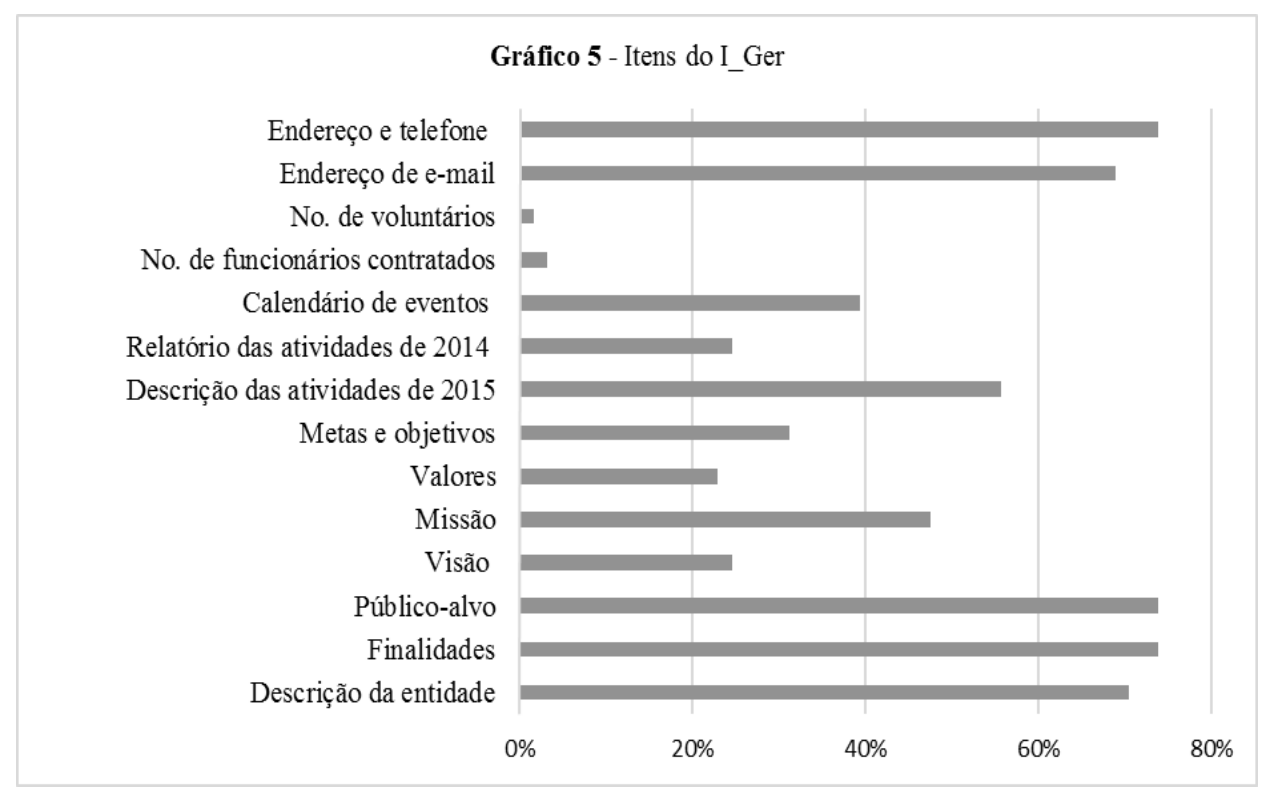

Fonte: Elaborado pelos autores com base na verificação dos 61 websites.

O Gráfico 5 demonstra que as informações gerais sobre o telefone e endereço; a finalidade da entidade; o público-alvo; a descrição da entidade; e o endereço de e-mail foram as mais presentes nos websites das entidades da amostra. Já o número de voluntários e o número de funcionários contratados raramente foram encontrados. Apenas $25 \%$ das entidades divulgaram relatórios das atividades desenvolvidas no ano de 2014, documento onde a entidade informa para a sociedade os projetos e programas realizados, o número de pessoas beneficiadas, além de outras informações relevantes para o melhor conhecimento de suas ações.

Gráfico 6 - Itens do I_FinGov

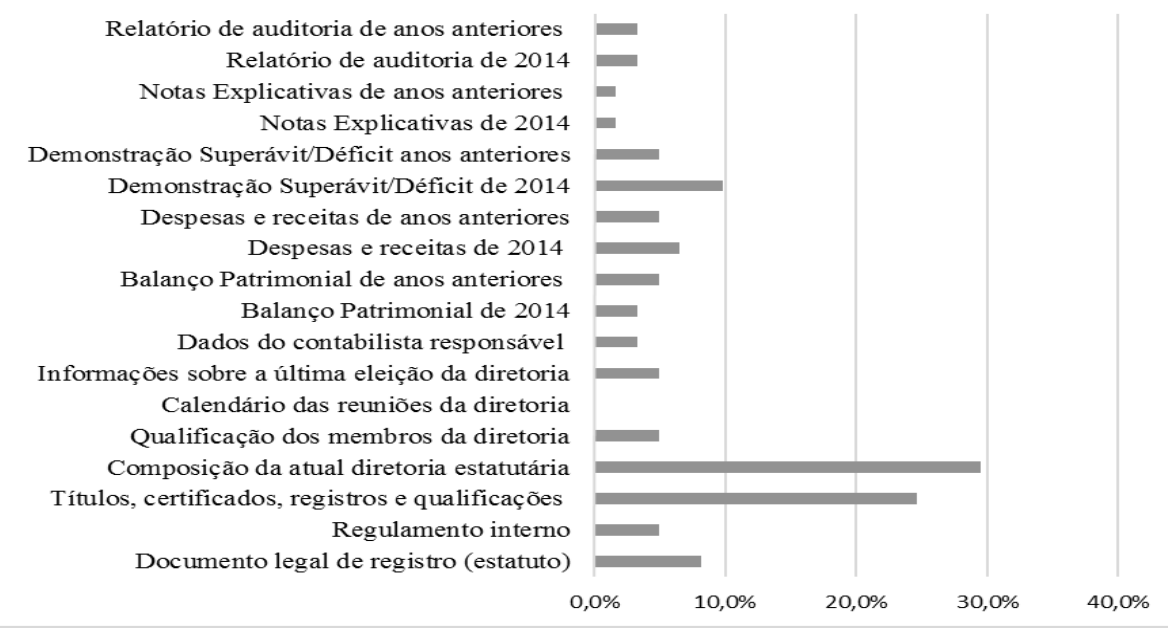

Fonte: Elaborado pelos autores com base na verificação dos 61 websites. 
O Gráfico 6 ilustra a preocupante situação encontrada na pesquisa sobre as informações financeiras e sobre governança das entidades, já que o item mais utilizado (composição da atual diretoria estatutária) teve menos de $30 \%$ de aderência. Com apenas $25 \%$ de aderência, o item títulos, certificados e registros que a entidade possui à nível federal, estadual e municipal ficou na segunda posição. Os demais itens do índice tiveram aderência abaixo de 10\%, incluindo as demonstrações contábeis, fato de relevante alerta.

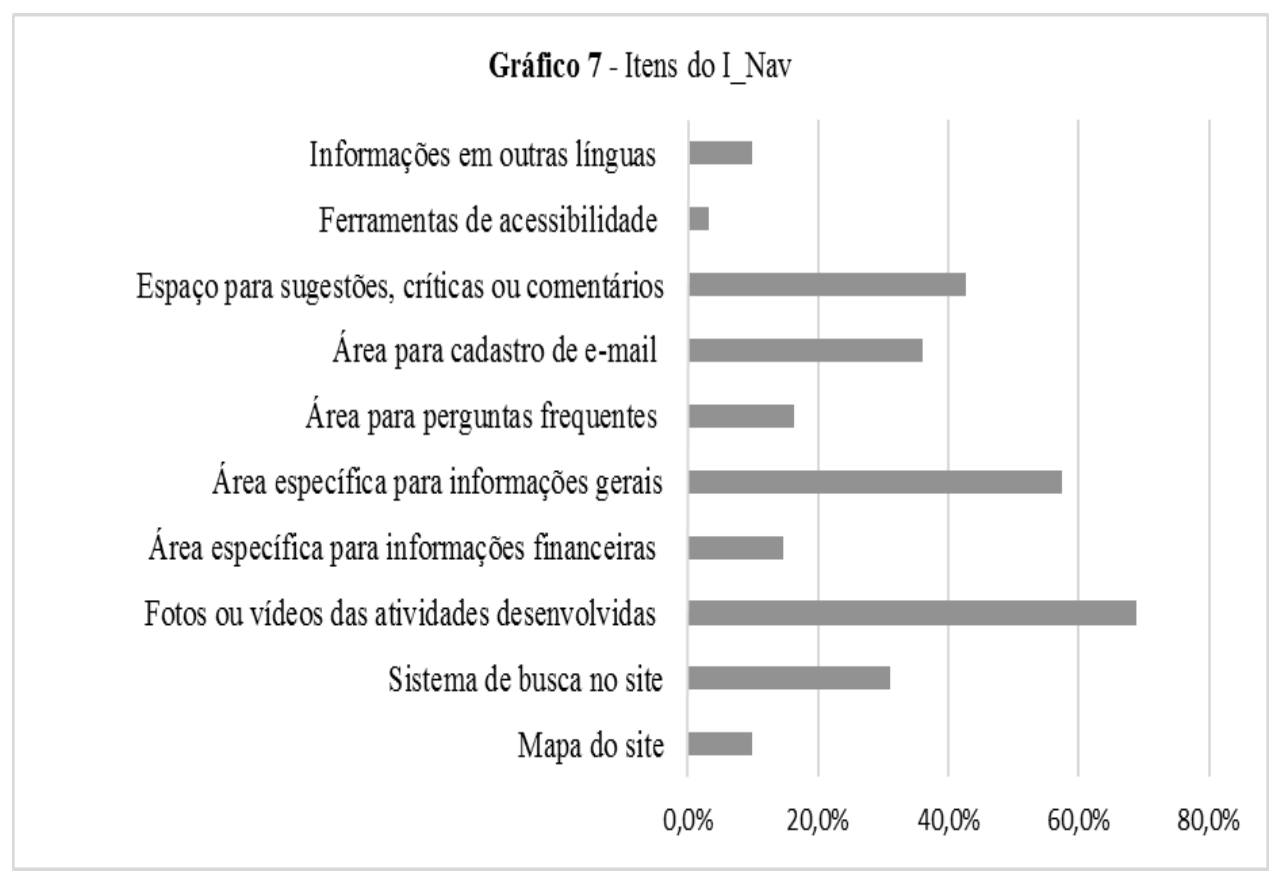

Fonte: Elaborado pelos autores com base na verificação dos 61 websites.

Quanto às ferramentas que contribuem para uma melhor navegabilidade dos websites das entidades, destacam-se no Gráfico 7 as fotos e vídeos das atividades desenvolvidas, com 69\% de aderência; área específica para informações gerais, com $57,4 \%$; e espaço para o envio de sugestões, críticas ou comentários, com $42,6 \%$ de aderência. Apenas $3 \%$ apresentaram alguma ferramenta de acessibilidade para pessoas com deficiências, não sendo encontrada a ferramenta até mesmo em entidades voltadas às pessoas com deficiência.

Diante desses resultados, percebe-se a grande necessidade de um avanço na transparência e accountability das entidades pesquisadas, como também a necessidade de uma maior exigência dos stakeholders por maiores informações disponibilizadas via website.

\section{3 ÍNDICE DE DISCLOSURE VIA WEBSITE E AS CARACTERÍSTICAS DAS ENTIDADES}

. Foram realizadas regressões simples para verificar a existência de significância estatística entre alguma das variáveis independentes diretamente na variável dependente I_DiscWeb. Dentre as cinco variáveis independentes, mostraram-se significativas a $10 \%$ as Receitas provenientes de Doações (Rdoa) e Tempo como UPF (Temp), conforme demonstra a Tabela 5. 
Tabela 5: Resumo dos Resultados das Regressões Simples

\begin{tabular}{ccccc}
\hline Variável Independente & R-quadrado & $\alpha$ & $\beta$ & $p$-valor \\
\hline Port & 0,004754 & 0,142332 & 0,007227 & 0,597516 \\
\hline Rdoa & 0,056173 & 0,195593 & 0,107465 & $0,065902^{*}$ \\
\hline Rgov & 0,018358 & 0,260165 & 0,080321 & 0,297816 \\
\hline Rent & 0,001015 & 0,245501 & 0,010168 & 0,807461 \\
\hline Temp & 0,048291 & 0,373097 & $0,088814^{*}$ \\
\hline
\end{tabular}

. Com os pressupostos da regressão simples testados e comprovados, confirmando a tendência à distribuição normal ( $p$-valor $=0,725227$ ) e à homocedasticidade ( $p$-valor $=0,227855)$, foi possível aceitar a segunda hipótese desta pesquisa a 10\% ( $p$-valor $=0,0659$ ), considerando que quanto maior a proporção de receitas por doações maior a quantidade de informações divulgadas pelas entidades do terceiro setor via website. Segundo o R-quadrado, essa característica da entidade explica $5 \%$ da variação no I_DiscWeb.

Esse resultado mostra-se preocupante já que o disclosure via website pode estar sendo utilizado apenas para a divulgação da imagem da entidade com objetivo de uma maior captação de recursos e não como ferramenta para a transparência e accountability. Tais achados corroboram com as pesquisas de Gandia (2011, p. 71), Saxton e Guo (2011, p. 285) e Saxton, Kuo e Ho (2012, p. 1069).

Também, com os pressupostos testados e comprovados, confirmando a tendência à distribuição normal ( $p$-valor $=0,555637$ ) e à homocedasticidade ( $p$-valor $=0,663035)$, foi possível aceitar a quinta hipótese da pesquisa a $10 \%$ ( $p$-valor $=0,0888$ ), que considera que quanto menor o tempo que a entidade possui como UPF, maior o I_DiscWeb. O R-quadrado de 0,048291 indica que essa característica das entidades da amostra pode explicar 4,8\% do I_DiscWeb.

Esses achados divergem da pesquisa de Gandia (2011, p. 67), contudo corroboram com as pesquisas de Saxton e Guo (2011, p. 285), Mendes-da-Silva et al. (2009, p. 194) e Mendes-da-Silva e Onusic (2010, p. 60). Isso indica que entidades mais jovens tendem a recorrer à tecnologia para melhorar a comunicação com seus stakeholders, diferentemente das entidades mais antigas.

No entanto, nota-se que o porte da entidade $(\mathrm{H} 1)$ e a rentabilidade $(\mathrm{H} 4)$ não tiveram influência significativa no I_DiscWeb, resultado contrário ao encontrado por Saxton, Kuo e Ho (2012, p. 1056). Já a proporção de receitas advindas do governo $(\mathrm{H} 3)$, que também não apresentou influência significativa no I_DiscWeb, vai de encontro aos achados de Lima e Pereira (2011, p. 28) e ratifica a aceitação da segunda hipótese.

\section{CONSIDERAÇÕES FINAIS}

Neste estudo buscou-se, primeiramente, examinar o nível de disclosure voluntário de informações financeiras e não financeiras das entidades do terceiro setor, por meio do Índice de Disclosure via Website e os seguintes índices parciais: Índice de Informações Gerais; Índice de Informações Financeiras e sobre Governança; Índice de Apresentação e Navegação do Website. Após esse exame, identificou-se a existência de associação entre o referido nível e as características das entidades estudadas.

A pesquisa revelou que as entidades possuem baixa preocupação com o disclosure voluntário, priorizando o disclosure obrigatório ao fornecer informações ao Ministério da Justiça, o que dificulta aos demais stakeholders, principalmente aos doadores e sociedade em geral, de praticarem a avaliação e o controle do desempenho das organizações. As entidades da amostra pouco se utilizam dos benefícios da Internet para a divulgação de informações financeiras e não financeiras, já que uma boa parte da 
amostra não possuía website e as entidades que possuíam apresentaram nível baixo, tanto no Índice de Disclosure via Website, quanto nos índices parciais, sendo o Índice de Informações Financeiras e sobre Governança o de menor nível.

Além disso, diante do baixo nível de disclosure de informações financeiras e sobre governança, pode-se apontar que entidades que dependem de doações utilizam-se da internet, principalmente, como estratégica de autopromoção diante de potenciais doadores e não como forma de melhorar a transparência de seus atos e, consequentemente, a governança e accountability, corroborando com as pesquisas de Saxton e Guo (2011, p. 279) e Saxton, Kuo e Ho (2012, p. 1057).

Também foi possível constatar que, independentemente do porte, do volume de recursos recebido por subvenções governamentais e da rentabilidade das organizações, a maioria das entidades estudadas não adota práticas de gestão profissionalizadas, já que não prezam pela comunicação com seus stakeholders. Esses achados também reforçam a possibilidade da existência de interesse estratégico na divulgação de informações apenas por parte das organizações que pretendem aumentar o volume recebido em doações, como visto anteriormente.

Foi possível verificar, ainda, a preocupação das organizações mais recentes em construir uma boa reputação e, assim, angariar a confiança dos stakeholders. Além disso, pode indicar uma tendência à inércia na administração das organizações mais antigas, por desprezarem a importância da transparência e da comunicação para o aumento da governança.

Sendo assim, reforça-se a necessidade de maior exigência por parte dos stakeholders e conscientização dos gestores das entidades do terceiro setor para que haja maior transparência, principalmente de informações financeiras e de governança, e melhor comunicação via website, incentivando e fortalecendo o controle social (Zuccolotto, 2015, p. 69) e reduzindo a assimetria informacional presente na relação "principal" (sociedade) e "agente" (entidades do terceiro setor) (Lima \& Pereira, 2011, p. 24).

Ressalta-se que o atual estudo pode servir como um guia de boas práticas para que as entidades do terceiro setor possam melhorar o conteúdo, a navegabilidade e a capacidade de comunicação com seus stakeholders por meio de seus websites. Se as recomendações forem implementadas, as entidades podem aumentar seu nível de disclosure e, assim, melhorar a transparência e accountability.

Importante destacar que as conclusões se restringem à amostra e ao período analisado. Para pesquisas futuras, sugere-se que sejam incluídas outras variáveis que possam influenciar no I_DiscWeb, inclusive indicadores de desempenho dos projetos sociais realizados pelas entidades. Além disso, sugere-se que sejam realizados estudos junto às entidades do terceiro setor com vistas a detectar os principais fatores impeditivos para o efetivo disclosure via website.

\section{REFERÊNCIAS}

Angonese, R., Sanches, J. R., \& Bezerra, F. A. (2014). Determinantes da divulgação de informações voluntárias por meio da internet. ConTexto, 14(26), 19-31.

Ávila, L. A. C. de. (2013). Governança corporativa, desempenho econômico-financeiro e volume de operações no terceiro setor: um estudo de caso na Fundação Rádio e Televisão Educativa de Uberlândia. Tese (Doutorado em Administração de Empresas) - Fundação Getúlio Vargas (FGV), São Paulo, SP, Brasil.

Behn, B. K. B., DeVries, D. D., \& Lin, J. (2010). The determinants of transparency in nonprofit organizations: An exploratory study. Advances in Accounting incorporating Advances in International Accounting, 26(4), 6-12. 
Borges, G. M. da C., Paulo, E., \& Pereira, J. M. (2014). Estudo sobre a Influência do Financiamento no Desempenho das Organizações do Terceiro Setor. V Congresso Nacional de Administração e Ciências Contábeis - AdCont 2014 (p. 1-16). Rio de Janeiro, RJ, Brasil.

Campos, G. M., Moreira, R. de L., \& Scalzer, R. S. (2014). Financial Reporting: Reflection On Transparency In The Third Sector. Sociedade, Contabilidade e Gestão, 9(3), 130-142.

Cruz, C. F., Ferreira, A. C. D. S., Silva, L. M. Da, \& Macedo, M. Á. D. S. (2012). Transparência da gestão pública municipal: um estudo a partir dos portais eletrônicos dos maiores municípios brasileiros. Revista de Administração Pública, 46(1), 153-176. Rio de Janeiro, RJ.

Dumont, G. E. (2013). Nonprofit Virtual Accountability: An Index and Its Application. Nonprofit and Voluntary Sector Quarterly, 42(5), 1049-1067.

Flach, L., Rosa, N. N. La, \& Stupp, D. R. (2014). Transparência online das informações de organizações do terceiro setor. Congresso UFSC de Controladoria e Finanças (p. 1-17). Florianópolis, SC, Brasil.

Gandia, J. L. (2011). Internet Disclosure by Nonprofit Organizations: Empirical Evidence of Nongovernmental Organizations for Development in Spain. Nonprofit and Voluntary Sector Quarterly, 40(1), 57-78.

Greenlee, J. S., \& Brown, K. L. (1999). The impact of accounting information on contributions to charitable organizations. Research in Accounting Regulation, 13, 111-125.

Guo, C. (2007). When Government Becomes the Principal Philanthropist: The Effects of Public Funding on Patterns of Nonprofit Governance. Public Administration Review, 67(3), 458-71.

Hair, J. F., Black, W. C., Babin, B. J., Anderson, R. E., \& Tatham, R. L. (2009). Análise multivariada de dados. São Paulo: Bookman Editora.

Ingenhoff, D., \& Koelling, A. M. (2009). The potential of Web sites as a relationship building tool for charitable fundraising NPOs. Public Relations Review, 35(1), 66-73.

Jamieson, D. (2009). Building relationships in the networked age: Some implications of the internet for nonprofit organizations. The Philanthropist, 15(2), 23-32.

Kang, S., \& Norton, H. E. (2004). Nonprofit organizations' use of the World Wide Web: Are they sufficiently fulfilling organizational goals? Public Relations Review, 30(3), 279-284.

Liket, K. C., \& Maas, K. (2015). Nonprofit Organizational Effectiveness: Analysis of Best Practices. Nonprofit and Voluntary Sector Quarterly, 44(2), 268-296.

Lima, E. M. L., \& Pereira, C. A. (2011). Associação entre indices de disclosure e características corporativas das instituições de ensino superior filantrópicas do Brasil-IESFB. Revista de Contabilidade e Organizações, 5(11), 23-48.

Mañas, A. V., \& Medeiros, E. E. (2012). Terceiro Setor : Um Estudo Sobre a sua Importância no Processo de Desenvolvimento Socio-Econômico. Perspectivas em Gestão \& Conhecimento, 2(2), 15-29.

Marudas, N., \& Jacobs, F. (2011). The effects of nonprofit organization-specific factors on governmental support to nonprofit organizations. Journal of Management \& Marketing Research, 8(1), 1-10.

Mendes-da-Silva, W., Ferraz-Andrade, J. M., Famá, R., \& Filho, J. A. M. (2009). Disclosure Via Website Corporativo: Um Exame De Informações Financeiras E De Governança No Mercado Brasileiro. Rae, 49(2), 190-205.

Mendes-Da-Silva, W., \& Onusic, L. M. (2014). Corporate e-disclosure determinants: Evidence from the Brazilian market. International Journal of Disclosure and Governance, 11(1), 54-73. Nature Publishing Group. 
Milani Filho, M. A. F. (2010). O Nível De Disclosure De Organizações Do Terceiro Setor: Uma Pesquisa Empírica Sobre As Entidades Filantrópicas Asilares Paulistas. Congresso Anpcont (Vol. 4, p. 1-16). Natal, RN, Brasil.

Nunes, R. D. S., \& Oliveira, A. M. V. D. (2004). Organizações do Terceiro Setor: uma tentativa de delimitação do universo de pesquisa. Anais do Encontro de Estudos Organizacionais-EnEO. Vitória, ES, Brasil.

O'Regan, K., \& Oster, S. (2002). Does Government Funding Alter Nonprofit Governance? Evidence from New York City Nonprofit Contractors. Journal of Policy Analysis and Management, 21(3), 359-379.

Olak, P. A., Slomski, V., \& Alves, C. V. O. (2008). As Publicações Acadêmicas Da Pesquisa Contábil No Brasil, No Âmbito Das Organizações Do Terceiro Setor. Revista de Educação e Pesquisa em Contabilidade, 2(1), 24-46.

Rezende, A. J., Facure, C. E. F., \& Dalmácio, F. Z. (2009). Práticas de Governança Corporativa em Organizações Sem Fins Lucrativos. Congresso USP de Controladoria e Contabilidade (p. 16). São Paulo, SP, Brasil.

Saxton, G. D., \& Guo, C. (2011). Accountability online: Understanding the Web-based accountability practices of nonprofit organizations. Nonprofit and Voluntary Sector Quarterly, 40(2), 270-295.

Saxton, G. D., Kuo, J.-S., \& Ho, Y.-C. (2012). The Determinants of Voluntary Financial Disclosure by Nonprofit Organizations. Nonprofit and Voluntary Sector Quarterly, 41(6), 1-21.

Tinkelman, D. (1999). Factors affecting the relation between donations to not-for-profit organizations and efficiency ratio. Research in Government and Nonprofit Accounting, 10, 135-161.

Trussel, J. M., \& Parsons, L. M. (2007). Financial Reporting Factors Affecting Donations to Charitable Organizations. Advances in Accounting, 23(7), 263-285.

Van Hulle, C., \& Dewaelheyns, N. (2014). Why do private non-profit organizations provide information on the Internet? Social Enterprise Journal, 10(1), 69-86.

Waters, R. D. (2007). Nonprofit organizations' use of the internet: A content analysis of communication trends on the internet sites of the philanthropy. Nonprofit Management and Leadership, 18(1), 59-76.

Zhuang, J., Saxton, G. D., \& Wu, H. (2014). Publicity vs. impact in nonprofit disclosures and donor preferences: a sequential game with one nonprofit organization and $\mathrm{N}$ donors. Annals of Operations Research, 221(1), 469-491.

Zuccolotto, R. (2015). Fatores determinantes da transparência do ciclo orçamentário estendido: evidências nos estados brasileiros. Tese (Doutorado em Ciências Contábeis) - Universidade de São Paulo, São Paulo. 